\section{Urinary Bladder Infection/Irritation among Female Users of a High-Tech Toilet}

Key words: recurrent cystitis, high-tech toilet

In 1988, a 50-year-old woman was diagnosed as having myelodysplastic syndrome (refractory anemia type) on the basis of pancytopenia, bone marrow erythroid hyperplasia, and trisomy 8 chromosomal abnormality. Since 1992, she had been dependent on blood transfusion to maintain her hemoglobin level above $6.0 \mathrm{~g} / \mathrm{dl}$. She developed acute cystitis in June 1993, 1 month after installation of a high-tech toilet in her home. Following this episode, cystitis occurred 17 times during the subsequent 5 years to May 1998. In contrast, she had 2 episodes of cystitis during the 5 years from 1988 to 1993 preceding the toilet renewal. From 1988 to 1998 , the leukocyte counts ranged from $2-3 \times 10^{3} / \mathrm{mm}^{3}$ with $30-50 \%$ neutrophils. The symptoms usually included frequency, dysuria, and suprapubic discomfort. Urinalysis confirmed the presence of pyuria in most episodes. Urine culture was performed on 3 occasions and 2 samples taken before the start of antimicrobial treatment grew Klebsiella pneumoniae. Urological examination revealed no anatomic abnormalities. The disease was treated with short-term antimicrobial therapy.

In Japan, more and more houses are installing a high-tech toilet equipped with a warm seat and a control panel of buttons. If you press the button after defecation, a toothbrushshaped squirt gun appears from the back of the bowl and begins shooting a stream of warm water onto the anus. Some women may also press the "bidet" button to wash the external genitalia. The pressure of the water stream is adjustable from strong to weak. Finally, you press the button that starts the blow-dryer and wipe off the remaining water with toilet paper.

It is to be noted that our myelodysplastic patient developed recurrent cystitis only after installation of a high-tech toilet. The infection could not be attributed to her leukopenia alone, since she had already been leukopenic for the previous 5 years, during which cystitis seldom occurred. We also treated another case of acute cystitis with proven pyuria in a 57 -year-old woman with no underlying disease. This was her first and only cystitis that occurred 5 months after renovation of her toilet. Moreover, we had an opportunity to interview or treat 3 more middle-aged women who suffered from recurrent cystitis/bladder irritation while being fervent users of a high-tech toilet. One of them was shown to have pyuria in all 3 episodes examined. All these 5 women, suspecting its disease connection or according to the advice of a physician, refrained from using the washing system and have since remained symptom-free for months to years. The present observation suggests that Japan's high-tech toilets are not without pitfalls for females whose urethra is more proximal to the anus and shorter than that of males. While cleansing the anus, some water spills over the perineum and vulva, and may presumably contaminate the urethra with enteric bacilli. A strong stream of water for bidet would facilitate the ascent of organisms from the introitus into the bladder. Thus, we have come to suspect that some female users of this "sophisticated" toilet, but not male users, are at risk of developing urinary tract infection. The symptoms may vary in accordance with the severity of the infection. Apparently, neither health care workers nor manufacturers are aware of the possible health hazard of this sort. From the prophylactic standpoint, female patients presenting with cystitis-like symptoms should be questioned whether they are high-tech toilet users. Furthermore, a large-scale survey is needed to determine the incidence and pathogenesis of this peculiar condition.

\section{Isao MiYoshI, Taeko MitsuOKA and Hirokuni TAGUCHI}

From the Third Department of Medicine, Kochi Medical School, Kochi Received for publication October 9, 2002; Accepted for publication February 3, 2003

Reprint requests should be addressed to Dr. Isao Miyoshi, the Third Department of Medicine, Kochi Medical School, Kochi 783-8505 